DOI: http://doi.org/10.4038/sljastats.v20i3.7967

Sri Lankan Journal of Applied Statistics, Vol. (20-3)

\title{
Bayesian Estimation of Bivariate Weighted Exponential Distribution
}

\author{
Dhanya Nair $\mathbf{R}^{1 *}$ and E.I Abdul Sathar ${ }^{2}$ \\ ${ }^{1}$ Department of Statistics, Govt. Arts College, Thiruvananthapuram, India. \\ ${ }^{2}$ Department of Statistics, University of Kerala, Thiruvananthapuram, India. \\ ${ }^{*}$ Corresponding Author: meeradhanya2007@gmail.com
}

Received: 25 ${ }^{\text {th }}$ April 2018 / Revised: 24 ${ }^{\text {th }}$ April 2019 / Published: $31^{\text {st }}$ December 2019

CIAppstat-SL2019

\begin{abstract}
In this paper, we consider Bayesian estimation for the parameters of bivariate weighted exponential Distribution based on generalized exponential distribution (BWEGE). The BWEGE has four parameters, two scale parameters and two shape parameters, which makes it more flexible to describe different types of real data than its sub model, the three-parameter bivariate weighted exponential distribution with weighted exponential marginal (BWE distribution). This four-parameter BWEGE has explicit joint probability density and distribution function. The Bayes estimators of the parameters under squared error loss function are obtained using the Lindley's approximation method due to the lack of explicit forms of estimators.
\end{abstract}

Keywords: Bivariate Weighted Exponential Distribution; Bivariate Bayesian Estimation; Quadratic Loss Function.

\section{Introduction}

A shape parameter may be introduced to an exponential distribution in many ways and the different methods result in variety of weighted exponential (WE) distributions. For example, the gamma distribution, and the generalized exponential distribution are different weighted versions of the exponential distribution. Gupta and Kundu (2009), introduced a shape parameter to an exponential model using the approach of Azzalini (1985), which resulted in a new class of WE distributions. This new WE model has the probability density function (PDF) whose shape is very close to the shape of the PDFs of Weibull, gamma, log-normal and generalized exponential distributions. Therefore, this model can be used as an alternative to any of these distributions. It was observed that the proposed weighted exponential distribution is very flexible and can be used quite effectively to analyze positively 
skewed data and censored data. Al-Mutairi et al (2011) considered a new bivariate absolute continuous distribution whose marginals are WE distributions and called this new bivariate distribution as the bivariate distribution with the weighted exponential marginals (BWE). This BWE has three parameters, two scale parameters and one shape parameter. It was observed that this three-parameter BWE distribution has several desirable properties and it can be used as an alternative to the several three-parameter absolute continuous bivariate distributions. Mahdavi, et al. (2017) introduced an additional parameter to the above distribution and constructed a new flexible model, the four parameter weighted bivariate exponential distribution and they named it as bivariate weighted exponential Distribution based on generalized exponential distribution (BWEGE). It has two scale parameters and two shape parameters, which makes it more flexible to describe different types of real data than its sub model, the three-parameter bivariate weighted exponential distribution with weighted exponential marginals (BWE distribution). Several properties of BWEGE distribution have been established by them. It was found that the joint probability density function (PDF) and the joint moment generating function (MGF) can be expressed in explicit forms. As the maximum-likelihood estimators (MLEs) of unknown parameters cannot be obtained in explicit forms, they were obtained by solving four nonlinear equations. For illustrative purposes, they have analyzed the real bivariate data set using the BWEGE model and it was observed that BWEGE provides better fit than BWE model. Moreover, the generation of random samples from the BWEGE is straightforward.

In this paper we consider the Bayesian estimators of the parameters of BWEGE. The Bayes estimators of the parameters under squared error loss function are obtained using the Lindley's approximation method due to the lack of explicit forms of estimators. The rest of the paper is organized as follows. In section 2 we presented the model. In Section 3 we derived the Bayesian estimators of the parameters of BWEGE. In Section 4 we calculated the proposed estimators for a numerical data.

\section{Bivariate weighted exponential distribution with weighted exponential marginals}

The random variable $X$ is said to have weighted exponential distribution with the shape and scale parameters as $\alpha>0$ and $\lambda>0$ respectively and denoted by $\operatorname{WE}(\alpha$, $\lambda)$, if it has the following PDF

$$
f_{X}(x ; \alpha, \lambda)=\frac{\alpha+1}{\alpha} \lambda e^{-\lambda x}\left(1-e^{-\alpha \lambda x}\right), \quad x \geq 0
$$


Note that the $\operatorname{WE}(\alpha, \lambda)$ can be obtained by Gupta and Kundu (2009) exactly the same way Azzalini (1985) obtained the skew- normal distribution from two independent and identically distributed (i.i.d) normal distributions. Suppose $X_{1}$ and $X_{2}$ are i.i.d $\exp (\lambda)$, i.e. an exponential distribution with mean $1 / \lambda$, then for $\alpha>0$ consider a new random variable $X=X_{1}$, if $\alpha X_{1} \geq X_{2}$. Then $X$ follows $\operatorname{WE}(\alpha, \lambda)$. It is observed that the $\operatorname{PDF}$ of $\operatorname{WE}(\alpha, \lambda)$ is unimodal, and it has increasing hazard rate for all values of $\alpha$.

Al-Mutairi et al (2011) introduced a three parameter absolute continuous bivariate distribution with weighted exponential marginal denoted by $\operatorname{BWE}\left(\lambda_{1}, \lambda_{2}, \lambda_{3}\right)$.

A bivariate random variable $\left(\mathrm{X}_{1}, \mathrm{X}_{2}\right)$ has the BWE distribution if the joint pdf of $\left(\mathrm{X}_{1}, \mathrm{X}_{2}\right)$ is as follows:

$f_{X_{1}, X_{2}}\left(x_{1}, x_{2}\right)=\frac{\lambda \lambda_{1} \lambda_{2}}{\lambda_{3}} e^{-\lambda_{1} x_{1}} e^{-\lambda_{2} x_{2}}\left(1-e^{-\lambda_{3} z}\right), \quad x_{1} \geq 0, x_{2} \geq 0$

where $z=\min \left\{x_{1}, x_{2}\right\}$ and $\lambda=\lambda_{1}+\lambda_{2}+\lambda_{3}$

Mahdavi, et al. (2017) introduced an additional parameter to the above distribution and constructed a new flexible model, the four parameter weighted bivariate exponential distribution. A bivariate random variable $\left(\mathrm{X}_{1}, \mathrm{X}_{2}\right)$ has the bivariate weighted exponential Distribution based on generalized exponential distribution (BWEGE) with positive parameters $\lambda_{1}, \lambda_{2}, \lambda, \theta$ if the joint pdf of $\left(\mathrm{X}_{1}, \mathrm{X}_{2}\right)$ is as follows:

$f_{X_{1}, X_{2}}\left(x_{1}, x_{2}\right)=\frac{\lambda_{1} \lambda_{2}}{\lambda B(\lambda, \theta+1)} e^{-\lambda_{1} x_{1}} e^{-\lambda_{2} x_{2}}\left(1-e^{-\alpha z}\right)^{\theta}, x_{1} \geq 0, x_{2} \geq 0$

where $z=\min \left\{x_{1}, x_{2}\right\}$ and $\alpha=\frac{\lambda_{1}+\lambda_{2}}{\lambda}$ and $B(a, b)=\int_{0}^{1} t^{a-1}(1-t)^{b-1} d t$ is the beta function.

Note that when $\theta=1$, BWEGE reduces to three parameter BWE.

The generation of random samples from the BWEGE is straightforward. The following result may be used to generate random samples from the BWED.

Result: Suppose $\mathrm{U}_{1} \sim \exp \left(\lambda_{1}\right), \mathrm{U}_{2} \sim \exp \left(\lambda_{2}\right)$ and $\mathrm{V} \sim \mathrm{BE}(\theta, \lambda+1, \alpha)$, and they are independently distributed, where $\mathrm{BE}$ is the beta exponential distribution introduced by Nadarajah and Kotz (2006). If we define $\mathrm{X}_{1}=\mathrm{U}_{1}+\mathrm{V}$ and $\mathrm{X}_{2}=\mathrm{U}_{2}+\mathrm{V}$, then

$\left(\mathrm{X}_{1}, \mathrm{X}_{2}\right) \sim \operatorname{BWEGE}\left(\lambda_{1}, \lambda_{2}, \alpha, \theta\right)$ with joint pdf

$f_{X_{1}, X_{2}}\left(x_{1}, x_{2}\right)=\frac{\lambda_{1} \lambda_{2}}{\lambda B(\lambda, \theta+1)} e^{-\lambda_{1} x_{1}} e^{-\lambda_{2} x_{2}}\left(1-e^{-\alpha z}\right)^{\theta}, \quad x_{1} \geq 0, x_{2} \geq 0$

where $z=\min \left\{x_{1}, x_{2}\right\}$ and $\lambda=\frac{\lambda_{1}+\lambda_{2}}{\alpha}$ and $B(a, b)=\int_{0}^{1} t^{a-1}(1-t)^{b-1} d t \quad$ is the beta function. 


\section{Bayesian estimators of the parameters of BWEGE}

In this section, we discuss the Bayesian estimators of the unknown parameters......We have bivariate sample of size ' $n$ ': $D=\left\{\left(x_{11}, x_{21}\right),\left(x_{12}, x_{22}\right), \ldots,\left(x_{1 n}, x_{2 n}\right)\right\}$ from BWEGE with pdf (3).

The likelihood function is obtained as

$L=l(\underline{\theta} \mid \underline{x})=\frac{\lambda_{1}^{n} \lambda_{2}^{n}}{\lambda^{n}[\beta(\lambda, \theta+1)]^{n}} e^{-\lambda_{1} Y_{1}} e^{-\lambda_{2} Y_{2}} e^{\theta \sum_{i=1}^{n} \log \left(1-e^{-z_{i} \alpha}\right)}$

The form of the likelihood suggests a prior for $\left(\lambda_{1}, \lambda_{2}, \lambda, \theta\right)$ with density

$g(\underline{\theta})=k \lambda_{1}^{p_{1}-1} \lambda_{2}^{p_{2}-1} e^{-\lambda_{1} m_{1}} e^{-\lambda_{2} m_{2}} e^{-\alpha_{0}(\lambda+1)} e^{-\beta_{0} \theta}[\beta(\lambda, \theta+1)]^{-\nu_{0}}$

Here $m_{1}, m_{2}, p_{1}, p_{2}, v_{o}, \alpha_{o}, \beta_{o}$ are the hyperparameters and are all positive. The symbol $\mathrm{k}$ stand for the normalising constant.

So that the posterior density turns out to be

$$
\begin{gathered}
f(\underline{\theta} \mid \underline{x}) \propto \lambda_{1}^{p_{1}{ }^{*}-1} \lambda_{2}^{p_{2}{ }^{*}-1} e^{-\lambda_{1} m_{1}{ }^{*}} e^{-\lambda_{2} m_{2}{ }^{*}} e^{-\alpha_{0} \lambda} e^{-\theta z_{\alpha}} \lambda^{-n}[\beta(\lambda, \theta+1)]^{-v} \\
\text { where } z_{\alpha}=\beta_{0}-\sum_{i=1}^{n} \log \left(1-e^{-z_{i} \alpha}\right) \\
m_{1}^{*}=m_{1}+Y_{1}, m_{2}{ }^{*}=m_{2}+Y_{2}, p_{1}^{*}=n+p_{1}, p_{2}^{*}=n+p_{2}, v=n+v_{o}
\end{gathered}
$$

If the R.H.S of (7) is denoted by $l_{N}$, then the Bayes estimator of a function $U=U\left(\lambda_{1}, \lambda_{2}, \lambda, \theta\right)$ under squared error loss function is the posterior mean and is given by

$$
\frac{\iiint \int_{\lambda_{1}} \int_{\lambda} u\left(\lambda_{1}, \lambda_{2}, \lambda, \theta\right) l_{N} d \lambda_{1} d \lambda_{2} d \lambda d \theta}{\iiint_{\lambda_{1}} \int_{\lambda_{2}} l_{\theta} l_{N} d \lambda_{1} d \lambda_{2} d \lambda d \theta}
$$

which cannot be obtained in closed form. Therefore, in such a situation, we resort to the use of a numeric integration technique such as Lindely's approximation. Lindely (1980) developed an approximate procedure for the evaluation of the ratio of two 
Bayesian Estimation of Bivariate Weighted Exponential Distribution

integrals. This procedure, applied to the posterior expectation of the function $U(\lambda)$

for a given $\underline{x}$, is

$E(U(\lambda) / \underline{x})=\frac{\int U(\lambda) e^{Q(\lambda)} d \lambda}{\int e^{Q(\lambda)} d \lambda}$, where $Q(\lambda)=l(\lambda)+\rho(\lambda)$

$l(\lambda)$ is the logarithm of the likelihood function and $\rho(\lambda)$ is the logarithm of the prior density of $\lambda$.

Using Lindely's approximation, $E(U(\lambda) / \underline{x})$ is approximately estimated by

$E(U(\lambda) / \underline{x})=U+\frac{1}{2} \sum_{i} \sum_{j}\left(u_{i j}+2 u_{i} \rho_{j}\right) \sigma_{i j}+\frac{1}{2} \sum_{i} \sum_{j} \sum_{k} \sum_{l} L_{i j k} \sigma_{i j} \sigma_{k l} u_{l}+$ terms of order $n^{-2}$ or smaller

For the four parameter case, Lindely's approximation leads to

$$
\begin{gathered}
E(U(\Theta) / \underline{x})=U(\Theta)+\frac{1}{2} \sum_{i=1}^{4} U_{i i} \sigma_{i i}+U_{12} \sigma_{12}+U_{13} \sigma_{13}+U_{14} \sigma_{14}+U_{23} \sigma_{23}+U_{24} \sigma_{24}+U_{34} \sigma_{34}+ \\
A_{1}\left(\rho_{1}+a_{1}\right)+A_{2}\left(\rho_{2}+a_{2}\right)+A_{3}\left(\rho_{3}+a_{3}\right)+A_{4}\left(\rho_{4}+a_{4}\right)
\end{gathered}
$$

Where

$$
\begin{gathered}
\Theta=\left(\lambda_{1}, \lambda_{2}, \lambda, \theta\right) ; \quad U_{i}=\frac{\partial U}{\partial \lambda_{i}} ; U_{i j}=\frac{\partial^{2} U}{\partial \lambda_{i} \partial \lambda_{j}}: \rho_{j}=\frac{\partial \rho}{\partial \lambda_{j}} ; L_{i j k}=\frac{\partial^{3} l}{\partial \lambda_{i} \partial \lambda_{j} \partial \lambda_{k}}, \\
a_{j}+\rho_{j}=\sigma_{11} L_{11 j}+\sigma_{22} L_{22 j}+\sigma_{33} L_{33 j}+\sigma_{44} L_{44 j}+\rho_{j}+ \\
\left.2\left(\sigma_{12} L_{12 j}+\sigma_{13} L_{13 j}+\sigma_{14} L_{14 j}+\sigma_{23} L_{23 j}+\sigma_{24} L_{24 j}+\sigma_{34} L_{34 j}\right)\right)
\end{gathered}
$$

and

$$
A_{j}=U_{1} \sigma_{j 1}+U_{2} \sigma_{j 2}+U_{3} \sigma_{j 3}+U_{4} \sigma_{j 4}
$$

for $j=1,2,3,4$.

From equations (5) and (6), we obtain logarithm of likelihood and logarithm of prior as follows: 
$l(\Theta)=\log L=-\lambda_{1} Y_{1}-\lambda_{2} Y_{2}+n \log \lambda_{1}+n \log \lambda_{2}-n \log \lambda-n \log \beta(\lambda, \theta+1)+\theta \sum_{i=1}^{n} \log \left[1-e^{-z_{i} \alpha}\right]$

$\rho(\Theta)=\log g(\underline{\theta})=-m_{1} \lambda_{1}-m_{2} \lambda_{2}+\left(p_{1}-1\right) \log \lambda_{1}+\left(p_{2}-1\right) \log \lambda_{2}-v_{o} \log \beta(\lambda, \theta+1)-\alpha_{o}(\lambda+1)-\beta_{0} \theta$

Then

$$
\begin{array}{ll}
\rho_{1}=-m_{1}+\frac{p_{1}-1}{\lambda_{1}} ; & \rho_{2}=-m_{2}+\frac{p_{2}-1}{\lambda_{2}} \\
\rho_{3}=-v_{o}[\psi(\lambda)-\psi(\lambda+\theta+1)]-\alpha_{o} ; & \rho_{4}=-v_{o}[\psi(\theta+1)-\psi(\lambda+\theta+1)]-\beta_{o}
\end{array} ;
$$

where $\psi(\mathrm{x})$ is the digamma function.

$$
\begin{aligned}
& L_{11}=\frac{-n}{\lambda_{1}^{2}}-\frac{\theta}{\lambda^{2}} \sum_{i=1}^{n} \frac{z_{i}^{2} e^{-z_{i} \alpha}}{\left(1-e^{-z_{i} \alpha}\right)^{2}} \quad, \quad L_{111}=\frac{2 n}{\lambda_{1}^{3}}-\frac{\theta}{\lambda^{3}} \sum_{i=1}^{n} \frac{z_{i}^{3} e^{-z_{i} \alpha}\left(e^{-2 z_{i} \alpha}-1\right)}{\left(1-e^{-z_{i} \alpha}\right)^{4}} \\
& L_{121}=L_{112}=-\frac{\theta}{\lambda^{3}} \sum_{i=1}^{n} \frac{z_{i}^{3} e^{-z_{i} \alpha}\left(e^{-2 z_{i} \alpha}-1\right)}{\left(1-e^{-z_{i} \alpha}\right)^{4}}=L_{221}, \\
& L_{141}=L_{114}=-\frac{1}{\lambda^{2}} \sum_{i=1}^{n} \frac{z_{i}^{2} e^{-z_{i} \alpha}}{\left(1-e^{-z_{i} \alpha}\right)^{2}}=L_{224} \\
& L_{131}=L_{113}=-\frac{\theta}{\lambda^{4}} \sum_{i=1}^{n} \frac{z_{i}^{2} e^{-z_{i} \alpha}\left(1-e^{-z_{i} \alpha}\right)\left\{\left[z_{i}\left(\lambda_{1}+\lambda_{2}\right)-2 \lambda\right]\left[1-e^{-z_{i} \alpha}\right]+2 z_{i}\left(\lambda_{1}+\lambda_{2}\right) e^{-z_{i} \alpha}\right\}}{\left(1-e^{-z_{i} \alpha}\right)^{4}}=L_{223}=L_{123} \\
& L_{22}=\frac{-n}{\lambda_{2}^{2}}-\frac{\theta}{\lambda^{2}} \sum_{i=1}^{n} \frac{z_{i}^{2} e^{-z_{i} \alpha}}{\left(1-e^{-z_{i} \alpha}\right)^{2}}, L_{222}=\frac{2 n}{\lambda_{2}^{3}}-\frac{\theta}{\lambda^{3}} \sum_{i=1}^{n} \frac{z_{i}^{3} e^{-z_{i} \alpha}\left(e^{-2 z_{i} \alpha}-1\right)}{\left(1-e^{-z_{i} \alpha}\right)^{4}} \\
& L_{33}=\frac{n}{\lambda^{2}}-n\left[\psi^{\prime}(\lambda)-\psi^{\prime}(\lambda+\theta+1)\right]-\frac{\theta\left(\lambda_{1}+\lambda_{2}\right)}{\lambda^{4}} \sum_{i=1}^{n} \frac{z_{i} e^{-z_{i} \alpha}\left\{\left[z_{i}\left(\lambda_{1}+\lambda_{2}\right)-2 \lambda\left(1-e^{-z_{i} \alpha}\right)\right\}\right.}{\left(1-e^{-z_{i} \alpha}\right)^{2}}
\end{aligned}
$$


Bayesian Estimation of Bivariate Weighted Exponential Distribution

$$
\begin{aligned}
& L_{44}=-n\left[\psi^{\prime}(\theta+1)-\psi^{\prime}(\theta+1+\lambda)\right], L_{444}=-n\left[\psi^{\prime \prime}(\theta+1)-\psi^{\prime \prime}(\theta+1+\lambda)\right], \\
& L_{442}=n \psi^{\prime \prime}(\lambda+\theta+1) \\
& L_{441}=L_{144}=0=L_{442}=L_{244}, \quad L_{124}=L_{142}=L_{241}=-\frac{1}{\lambda^{2}} \sum_{i=1}^{n} \frac{z_{i}^{2} e^{-z_{i} \alpha}}{\left(1-e^{-z_{i} \alpha}\right)^{2}} \\
& L_{134}=L_{143}=L_{341}=\sum_{i=1}^{n} z_{i} e^{-z_{i} \alpha}\left(z_{i} \alpha-1+e^{-z_{i} \alpha}\right)=L_{342}=L_{243}=L_{234}, \quad \sigma_{i j} \text { are }
\end{aligned}
$$

obtained using $L_{i j}$

In Equation (11), choose $U=\lambda_{1}, U=\lambda_{2}, U=\lambda$ and $U=\theta$ respectively to obtain the Bayes estimators of $\lambda_{1}, \lambda_{2}, \lambda$ and $\theta$ under squared error loss function. Thus Equation (11) reduces to

$$
\begin{aligned}
& E\left(\lambda_{1} / \underline{x}\right)=\lambda_{1}+\sigma_{11}\left(\rho_{1}+a_{1}\right)+\sigma_{21}\left(\rho_{2}+a_{2}\right)+\sigma_{31}\left(\rho_{3}+a_{3}\right)+\sigma_{41}\left(\rho_{4}+a_{4}\right) \\
& E\left(\lambda_{2} / \underline{x}\right)=\lambda_{2}+\sigma_{12}\left(\rho_{1}+a_{1}\right)+\sigma_{22}\left(\rho_{2}+a_{2}\right)+\sigma_{32}\left(\rho_{3}+a_{3}\right)+\sigma_{42}\left(\rho_{4}+a_{4}\right) \\
& E(\lambda / \underline{x})=\lambda+\sigma_{13}\left(\rho_{1}+a_{1}\right)+\sigma_{23}\left(\rho_{2}+a_{2}\right)+\sigma_{33}\left(\rho_{3}+a_{3}\right)+\sigma_{43}\left(\rho_{4}+a_{4}\right) \\
& E(\theta / \underline{x})=\theta+\sigma_{14}\left(\rho_{1}+a_{1}\right)+\sigma_{24}\left(\rho_{2}+a_{2}\right)+\sigma_{34}\left(\rho_{3}+a_{3}\right)+\sigma_{44}\left(\rho_{4}+a_{4}\right)
\end{aligned}
$$

where all the functions at the R.H.S are to be evaluated at the MLE's.

\section{Data analysis}

In this section, we consider a real-data example from Al-Mutairi et al. (2011) which consist of scores from 25 first graduate students in Probability-I and Inference-I of a premier Institute in India. The data set is presented as follows:

$X 1: 53,55,85,87,22,23,25,93,51,62,53,32,43,47,30,88,59,49,42,71,41$, $82,75,93,37$

$X 2: 89,90,59,50,25,29,54,62,39,25,89,32,33,63,38,77,55,41,31,66,57$, $32,43,88,34$

Since the smallest values of Akaike information criterion (AIC) and Bayesian information criterion (BIC) are obtained for BWEGE distribution, Mahdavi, et al. (2017) fitted the same data to the BWEGE and obtained the MLE of the parameters 
and we used the same estimated values for deriving our Bayesian estimators. Using equations (14) -(17) we derived the Bayesian estimators for the same data and are reported in Table 1.

Table 1. Estimated parameters for the score data set

\begin{tabular}{|llllll|}
\hline Parameters & $\lambda_{1}$ & $\lambda_{2}$ & $\lambda$ & $\theta$ \\
\hline MLE & & & & \\
\hline & 0.0485 & 0.0597 & 216.4 & 1 \\
\hline Bayesian & & & & & \\
\hline
\end{tabular}

\section{Comparison between MLE and Bayesian estimators}

Monte Carlo simulation method is used out to assess the performance of MLE and Bayesian estimator. The BWEGE distribution with parameter $\lambda_{1}=0.08, \lambda_{2}=0.09, \lambda$ $=230$ and $\theta=1$ is used for the simulation. The bias and mean squared error (MSE) of the estimators are calculated and the obtained values are presented in Table 2. It is observed that the MSE of the Bayesian estimator was less as compared to MLE. Hence Bayesian estimator is preferred to MLE.

Table 2(a). Bias and MSE (in brackets) of MLE

\begin{tabular}{|llll|}
\hline 0.06747 & 0.09230 & 0.20651 & 0.35225 \\
\hline$(0.00610)$ & $(0.01079)$ & $(0.05587)$ & $(0.14337)$ \\
\hline
\end{tabular}

Table 2(b). Bias and MSE (in brackets) of Bayesian estimator

\begin{tabular}{|cccc|}
\hline 0.02730 & 0.03548 & 0.08023 & 0.15520 \\
\hline$(0.00108)$ & $(0.00184)$ & $(0.00845)$ & $(0.03652)$ \\
\hline
\end{tabular}

\section{References}

1. Al-Mutairi, D., Ghitany, M., and Kundu, D. (2011). A new bivariate distribution with weighted exponential marginals and its multivariate generalization. Stat. Pap., 52(4), 921-936. 
Bayesian Estimation of Bivariate Weighted Exponential Distribution

2. Azzalini, A. (1985). A class of distributions which includes the normal ones. Scand. J. Stat., 12(2), 171-178.

3. Gupta, R. D. and Kundu, D. (2009). A new class of weighted exponential distributions. Statistics, 43(6), 621-634.

4. Lindley, D. V. (1980). Approximate Bayesian methods. In Bayesian Statistics (eds. J.M. Bernardo, M.H. DeGroot, D.V. Lindley, A.F.M. Smith), Valencia: University Press.

5. Mahdavi, A., Fathizadeh, M. and Jamalizadeh, A. (2017). On the bivariate weighted exponential distribution based on the generalized exponential distribution. Communications in Statistics. Theory and Methods 46(8), 36413648 .

6. Nadarajah, S. and Kotz, S. (2006). The beta exponential distribution. Reliab. Eng. Syst. Saf. 91(6), 689-697. 